\title{
Primeros datos sobre movimientos de cigüe- ñas blancas Ciconia ciconia L., 1758 anilladas como pollos en nido en Navarra: 2012-2015.
} Initial data concerning movements of White Storks Ciconia ciconia L., 1758 ringed as nestlings in Navarre: 2012-2015.

\author{
Jaime Resano-Mayor ${ }^{1}$, Juan María Barbarín², Daniel Alonso², Blanca Fernández-Eslava², \\ Diego Villanua², Jesús Mari Lekuona ${ }^{3}$, Ricardo Rodríguez ${ }^{4}$, Juan Arizaga²
}

$\xi^{*}$

\begin{abstract}
Resumen
En las últimas décadas, la población reproductora de cigüeña blanca Ciconia ciconia L., 1758 en Navarra ha aumentado exponencialmente. La abundancia de comida predecible en vertederos, que proporcionan uno de los recursos tróficos más utilizados por la especie, parece haber tenido influencia tanto a nivel demográfico como en la distribución espacio-temporal en el conjunto de la península ibérica. Con el objetivo de estudiar los patrones de dispersión, movimientos y usos del hábitat de la población de cigüeñas en Navarra, durante el periodo 2012-2015 se anillaron 243 pollos en 9 colonias de cría del sur de Navarra. De todos ellos, el $16 \%$ de los individuos $(n=37)$ fue observado con posterioridad, tanto vivos y en buen estado (i.e., avistamientos) como muertos (i.e., recuperaciones, en su mayoría por electrocución). Todos los avistamientos, excepto tres, se realizaron en vertederos $(n=64)$, en donde el esfuerzo de muestreo fue considerablemente mayor. Dos avistamientos correspondieron a cigüeñas observadas en humedales, así como otro ejemplar que se observó dos años después en la colonia donde nació (i.e., reclutamiento). La distancia promedio de avistamiento en relación a la colonia
\end{abstract} \footnotetext{
Baltzerstrasse 6, 3012 Bern, Switzerland

2 Sociedad de Ciencias Aranzadi. Departamento de Ornitología Zorroagagaina 11 • E-20014 Donostia-S. Sebastián, Spain

3 Plaza del Soto 29 bajo, 31016 Pamplona, Spain

4 Gorosti Natural Sciences Society

Caldereria 34, E-31001 Pamplona, Spain

1 University of Bern. Division of Conservation Biology, Institute of Ecology and Evolution 
de anillamiento fue de 48 km (distancia máxima, 410 km). La mayoría de avistamientos/recuperaciones se realizó durante la primavera y verano, con independencia de la edad. Todas las aves que se hallaron muertas $(n=7)$ fueron jóvenes encontrados en la proximidad de sus coIonias de nacimiento.

Palabras clave: Colonias, dispersión, electrocución, hábitat, vertederos.

\begin{abstract}
In recent decades, the breeding population of White Stork Ciconia ciconia L., 1758 in Navarre has increased exponentially. The abundance of predictable food in landfills, providing one of the most used food resources for the species, seems to have influenced both demographically and spatiotemporal distribution in the whole Iberian Peninsula. In order to study dispersal patterns, movements and habitat use of the White Stork population in Navarre, during the period 2012-2015, we ringed 243 chicks in 9 breeding colonies in southern Navarre. Of these, 16\% of the individuals ( $n=37$ ) were observed afterwards both alive and in good condition (i.e., sightings) and dead (i.e., recoveries), mostly by electrocution. All the sightings, except three, were made in landfills ( $n=61$ ), where the sampling effort was considerably higher. Two sightings corresponded to storks observed in wetlands, and another individual was observed two years after at the colony where it was born (i.e., recruitment). The average sighting distance in relation to the colony was $48 \mathrm{~km}$ (maximum distance, $410 \mathrm{~km}$ ). Most sightings/recoveries were obtained during the spring and summer, regardless of age. All dead birds $(n=7)$ were fledglings found in the vicinity of the colonies where they were born.
\end{abstract}

Key words: Colonies, dispersion, electrocution, habitat, landfills.

\title{
Laburpena
}

Azken hamarkadetan Nafarroako amiamoko zuri Ciconia ciconia L., 1758 populazio ugaltzaileak gora egin du modu esponentzialean. Zabortegietako janari ugariren aurreikuspenak, zeina espeziearen elikagai-baliabide garrantzitsuenetakoa baita, demografian zein iberiar penintsula osoko denbora-espazioko banaketan eragina izan du. Nafarroako amiamoko zuriaren populazioaren banaketa patroiak, mugimenduak eta habitat erabilera ikertzeko asmoz 2012 eta 2015 urteen artean Nafarroako hegoaldeko 9 ugaltze-koloniatan 243 txita eraztundu ziren. Horien \%16 ( $n=37$ ) ondoren behatu egin ziren; bai bizirik eta egoera onean zeudenak (behaketak) eta bai hilik (berreskurapenak, gehienak elektrokuzioz hilda). Hiru izan ezik, behaketa guztiak zabortegietan egin ziren $(n=64)$; horietan laginketa lana handiagoa izan zen. Bi amiamoko hezeguneetan behatu ziren eta beste ale bat jaio zeneko kolonia berean ikusi zen (errekrutatzea). Kolonia eta behaketa puntuen arteko batezbesteko distantzia 48 km-koa izan zen (distantzia gehienez, 410 km). Behaketa/berreskurapen gehienak udaberrian eta udaran egin ziren, adinari erreparatu gabe. Hilik aurkitu ziren hegazti guztiak $(n=7)$ ale gazteak izan ziren, beren jaiotze koloniatik gertu.

Gako hitzak: koloniak, sakabanaketa, elektrokuzioa, habitata, zabortegiak. 


\section{Introducción}

El uso por las aves de recursos tróficos de origen humano, abundantes y predecibles temporal y espacialmente, es un fenómeno conocido, particularmente en especies de carácter oportunista. Tal es el caso de varias especies de gaviotas, garzas y cigüeñas en relación a los vertederos (Tortosa et al., 2002; Ramos et al., 2009; Arizaga et al., 2013). Los efectos de estos recursos sobre diversos aspectos de la biología y ecología de dichas especies, entre ellos la tendencia de crecimiento de sus poblaciones así como los patrones de movimiento y distribución espacial, tienen interés tanto desde un punto de vista científico como administrativo (gestión de especies y poblaciones) (Blanco, 1996; Tortosa et al., 2002; Arizaga et al., 2014)

Durante las últimas dos décadas, la población reproductora de cigüeña blanca Ciconia ciconia L., 1758 en España ha aumentado notablemente. El último censo realizado a nivel nacional en 2004 estimó el número de parejas en 33.217 (Molina \& Del Moral, 2005). En Navarra, el número de parejas se estimó en 621, lo que supuso un incremento superior al $550 \%$ respecto al anterior censo de 1994, convirtiéndola en la provincia con un mayor aumento poblacional (Molina \& Del Moral, 2005). Desde 2004, la tendencia al alza de la población de cigüeñas en Navarra ha continuado, aunque en los últimos años el crecimiento se ha estabilizado, habiéndose censado 889 parejas en 2012, 914 en 2013, 934 en 2014 y 952 en 2015 (Gurelur, 2015). La adaptación por parte de las cigüeñas a explotar vertederos abiertos, junto al aumento en la producción de residuos urbanos, parecen haber contribuido en gran medida al incremento de las poblaciones ibéricas y haber afectado a sus hábitos migratorios (Sanz-Aguilar et al., 2015).

Paralelamente al aumento de la población reproductora, en las últimas décadas se ha detectado un incremento en el número de ejemplares invernantes en toda España [+312\% entre 1996 y 2004 (Molina \& Del Moral, 2005)]. Una parte de las cigüeñas que invernan en España corresponde a aves de origen ibérico y otra a ejemplares procedentes de la población occidental europea, en su mayoría de Alemania, Holanda y Francia (Vergara et al., 2004; Molina \& Del Moral, 2005). Estos censos invernales, en consecuencia, reflejan un cambio en la conducta migratoria de las poblaciones en Europa occidental (Flack et al., 2016). Aunque la población invernante se concentra mayoritariamente en el suroeste peninsular, en el norte su número también ha aumentado, distribuyéndose principalmente a lo largo del valle del Ebro. Según clases de edad, las aves que no cruzan el Sahara son, principalmente, adultos (Molina \& Del Moral, 2005), mientras que las que se desplazan hasta las áreas de invernada en África subsahariana son, principalmente, juveniles (Flack et al., 2016).

En este escenario, en 2012 comenzó en Navarra un proyecto de marcaje de pollos de cigüeña blanca con el objetivo general de estimar diferentes parámetros de la población, tales como patrones de dispersión y movimiento, ecología espacial y parámetros demográficos, entre otros. Hasta entonces, solo 9 pollos habían sido anillados en la población navarra de cigüeña blanca (fuente: oficinas de anillamiento de Aranzadi e ICONA). Tras cuatro años de anillamiento, este artículo describe, por primera vez para Navarra, los patrones 
de dispersión y movimientos, así como la tipología de hábitats utilizados por los individuos marcados, en su mayoría pertenecientes a la fracción no reproductora (inmaduros) de la población.

\section{Material y métodos}

\section{Área y periodo de estudio}

Durante el periodo 2012-2015 se controlaron 9 colonias de cría de cigüeña blanca en el sur de Navarra: Azagra, Castejón, Corella, Cortes, Funes, Milagro, San Adrián, Tudela y Villafranca (Fig. 1). En su conjunto, éstas incluyeron nidos construidos en riscos, árboles o soportes similares, iglesias u otros edificios, torretas de luz o grúas de obra. El seguimiento de cada colonia comenzó en los meses de marzo y abril, durante el periodo de incubación, con el fin de estimar el número de nidos con puesta y su accesibilidad para el posterior marcaje de los pollos. En este sentido, se consideraron únicamente aquellos nidos cuyo acceso resultaba seguro, teniendo en cuenta tanto la seguridad de los pollos como de las personas encargadas de su anillamiento. En mayo, se realizó una segunda visita para comprobar el número de nidos con pollos y estimar la edad de los mismos en base al desarrollo de sus plumas. El día del anillamiento de los pollos, cada nido recibió un código identificativo el primer año en que se anillaron pollos en él, de cara a su identificación en años sucesivos. Para el acceso a los nidos se utilizó material de escalada (e.g. arnés, cuerdas y casco) así como una escalera en aquellos nidos situados en árboles o edificaciones. En el caso de nidos situados en iglesias o edificios particulares, se contó con el consentimiento de párrocos y propietarios.

\section{Anillamiento y seguimiento}

Durante los meses de mayo y junio se realizó el anillamiento de los pollos cuando tenían una edad de 25 a 50 días (Barbraud et al., 1999; Aguirre, 2006). En total, durante el periodo 2012-2015 se anillaron 37, 65, 82 y 59 pollos, respectivamente. A cada pollo se le colocó una anilla metálica convencional con remite "Aranzadi" en tibia así como una anilla de PVC blanca con cuatro dígitos alfanuméricos negros, en la tibia de la pata opuesta. El código utilizado fue publicado en cr-birding (http://www.cr-birding.org/node/194) y se coordinó con el resto de los proyectos de marcaje de cigüeña blanca en Europa, para evitar solapamiento de códigos.

Tras el abandono de las colonias por los pollos marcados, se recopiló información relativa a ejemplares observados e identificados a través de la anilla de PVC (avistamientos). Tales avistamientos se llevaron a cabo principalmente en cultivos, zonas húmedas y, sobre todo, en vertederos, tanto por observadores del propio equipo de marcaje como por observadores que, a título voluntario, comunicaron sus avistamientos. Especial esfuerzo de avistamiento se realizó en los vertederos de Culebrete (Navarra) y Ejea de los Caballeros 
(Zaragoza). Durante el periodo 2012-2015, se realizó al menos una visita quincenal a cada vertedero. La mayor parte de las visitas se realizaron a media mañana (10:00-11:00) y duraron entre 2-4 horas. Las visitas se realizaron con un vehículo todoterreno y todos los censos y observaciones se realizaron desde el interior del vehículo para evitar molestias a las aves. La localización y lectura de ejemplares anillados se realizó mediante el uso de prismáticos, telescopio terrestre y cámaras fotográficas réflex con objetivos de diferente alcance. Una vez revisado completamente un grupo de cigüeñas se continuaba con la búsqueda de otros grupos, hasta haber revisado todos los diferentes grupos presentes dentro del recinto. Por otro lado, también se hizo un muestreo periódico (quincenal) en los arrozales del entorno de Arguedas (Navarra). El resto de citas, tanto en la península como en el sur de Francia se realizaron por particulares que, habiendo observado individuos anillados, lo comunicaron a alguna oficina de anillamiento. Asimismo, también se utilizó en este artículo información procedente de aves halladas muertas (recuperaciones). En este trabajo se recogen datos recopilados entre el 03.08.2012 y el 09.12.2015.

\section{Resultados}

En total, obtuvimos 71 avistamientos/recuperaciones de 38 individuos (Fig. 1). Esto supone un 16\% de los ejemplares marcados durante el periodo de estudio (2012-2015). El 82\% $(n=31)$ de los individuos fue avistado con vida y en buen estado, mientras que el $18 \%$ restante $(n=7)$ se recuperó muerto o herido de gravedad. La distancia máxima de avistamiento fue de 410 km: dos ejemplares anillados en Azagra en 2012 y 2014 y avistados en 2015 en sendos vertederos en Girona y Ciudad Real, respectivamente (Fig. 1). En promedio, la distancia de avistamiento/recuperación fue de $48 \mathrm{~km}(\mathrm{SE}=12 \mathrm{~km})$. La principal causa de mortalidad se debió a electrocución en líneas eléctricas o puntos de alimentación de corriente en catenarias de tren $(n=4)$. El 95\% ( $n=61)$ de los avistamientos fue en vertederos, siendo el de Culebrete el más frecuentado ( $n=52$; Fig. 1). Otros vertederos utilizados por las cigüeñas anilladas en Navarra fueron: Almagro (Ciudad Real; $n=4$ ), Ejea de los Caballeros (Zaragoza; $n=3$ ), Zaluaga (región de Aquitania, Francia; $n=1$ ) y Pedret i Marzá (Girona; $n=1$; Fig. 1). Sólo dos de las cigüeñas avistadas en áreas de alimentación fueron detectadas fuera de vertederos: una antigua gravera restaurada con humedales situada a tan sólo 20 km de Madrid (El Porcal; $n=1$ ) y un campo de arroz inundado en la Ribera de Navarra (Tudela; $n=1$ ).

Los avistamientos/recuperaciones tuvieron lugar a lo largo de todo el año, excepto en enero, octubre y noviembre, aunque la mayoría se realizaron durante los meses de primavera y verano (Fig. 2). Por años, el número de avistamientos/recuperaciones fue muy inferior en $2012(n=1)$ y $2013(n=7)$ en comparación a $2014(n=31)$ y $2015(n=32)$. Durante todo este periodo, la edad de los individuos avistados incluyó jóvenes del año $(n=34)$, así como aves en su segundo $(n=14)$, tercer $(n=16)$ y cuarto $(n=7)$ año calendario de 
Fig. 1.-Localización de las colonias en donde se anillaron pollos de cigüeña blanca en la ribera del Ebro (Navarra) y lugares donde se hallaron individuos anillados, tanto vivos como muertos. A) Colonias de anillamiento (cuadros blancos) y lugares próximos (puntos rojos) de avistamiento/recuperación de aves anilladas en esas colonias; el vertedero de Culebrete se señala con una flecha. Colonias: San Adrián (1), Azagra (2), Funes (3), Villafranca (4), Milagro (5), Castejón (6), Corella (7), Tudela (8) y Cortes (9). B) Mapa de la península ibérica y sur de Francia con todos los lugares donde se obtuvieron uno o más avistamientos/recuperaciones de cigüeñas anilladas en Navarra (colonias de la figura A). El cuadro de la figura $B$ corresponde al área representada en la figura $A$.

Fig. 1.- Site of the colonies in which White Stork chicks were ringed in the Ribera del Ebro (Navarre) and the locations in which ringed individuals were found, both alive and dead. A) Ringing colonies (white squares) and nearby sighting/recovery locations of birds ringed in these colonies (red dots); the Culebrete landfill is indicated with an arrow. Colonies: San Adrián (1), Azagra (2), Funes (3), Villafranca (4), Milagro (5), Castejón (6), Corella (7), Tudela (8) and Cortes (9). B) Map of the Iberian Peninsula and Southern France with all the locations in which one or more sightings/recoveries of storks ringed in Navarre were obtained (colonies in Figure A). The diagram in Figure B corresponds to the area represented in Figure A.

Fig. 2.- Representación a lo largo del año (enero-diciembre) del número de avistamientos (ejemplar vivo $(\mathrm{n}=$ 64): gris) y recuperaciones (ejemplar muerto $(n=7)$ : negro) de cigüeñas blancas marcadas como pollos en Navarra (periodo 2012-2015). En algunos casos el mismo individuo se avistó en más de una ocasión.

Fig. 2.- Representation throughout the year (January-December) of the number of sightings (live individual $(n=64)$ : grey) and recoveries (dead individual ( $n=7)$ : black) of White Storks ringed as chicks in Navarre (2012-

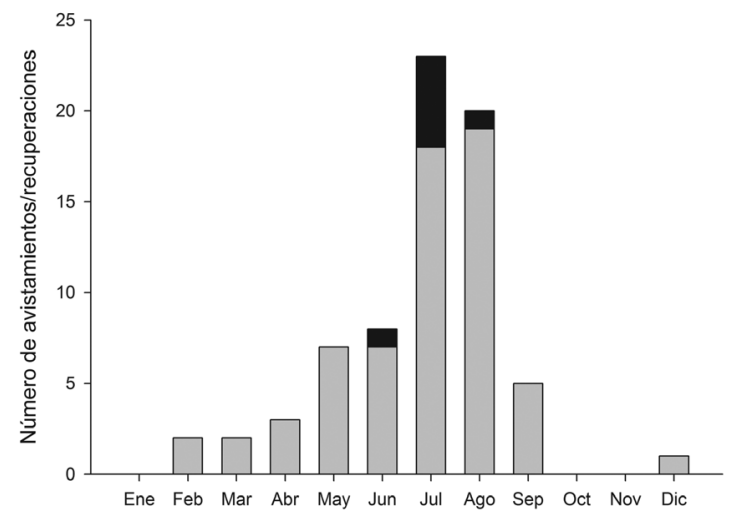

2015). In some cases the same individual was seen on more than one occasion. 


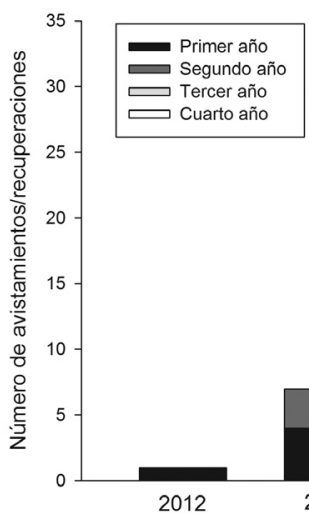

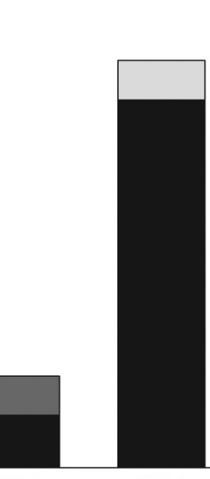

2013

2014

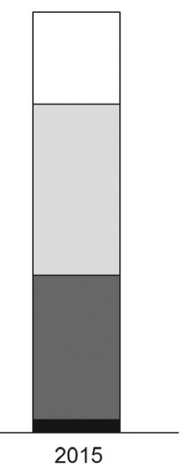

Fig. 3.- Número de avistamientos/recuperaciones por año (2012-2015). Los diferentes colores indican la edad (año calendario) de los individuos avistados/recuperados. En algunos casos el mismo individuo se avistó en diferentes años, es decir, con diferente edad.

Fig. 3.- Number of sightings/recoveries per year (2012-2015). The different colours indicate the age (calendar year) of the individuals seen/recovered. In some cases, the same individual was seen in different years, in other words, with different ages.

vida (Fig. 3). Por el contrario, todas las cigüeñas recuperadas heridas o muertas fueron jóvenes volantones, de 8 a 14 semanas de edad, halladas en la proximidad de sus colonias de nacimiento. La mayoría de los avistamientos pertenecieron a cigüeñas anilladas en Azagra $(n=31)$ y Corella $(n=26)$, seguido de Castejón $(n=3)$, Cortes $(n=2)$, Funes $(n=1)$ y Tudela $(n=1)$. Sin embargo, casi la mitad de los individuos hallados heridos o muertos se anillaron en la colonia de Castejón ( $n=3)$, situada junto a las vías del tren; el resto en Azagra $(n=1)$, Corella $(n=1)$, Funes $(n=1)$ y Villafranca $(n=1$; Tabla 1$)$

\begin{tabular}{|lcccc|}
\hline Colonia & Periodo & Pollos anillados & Avistamientos & Recuperaciones \\
\hline Azagra & $2012-2015$ & 105 & $31(14)$ & 1 \\
Castejón & $2013-2014$ & 19 & $3(2)$ & 3 \\
Corella & $2012-2015$ & 59 & $26(12)$ & 1 \\
Cortes & $2013-2015$ & 37 & $2(1)$ & 0 \\
Funes & $2013-2014$ & 6 & 1 & 1 \\
Milagro & 2013 & 3 & 0 & 0 \\
San Adrián & $2012-2013$ & 7 & 0 & 0 \\
Tudela & $2013-2014$ & 4 & 1 & 0 \\
Villafranca & 2014 & 3 & 0 & 1 \\
\hline Total & $2012-2015$ & 243 & $64(31)$ & 7 \\
\hline
\end{tabular}

Tabla 1.- Colonias de estudio de cigüeña blanca en Navarra y periodo (años) en que se anillaron pollos. Para cada colonia se detalla el número de pollos anillados y el número de avistamientos (ejemplar vivo) y recuperaciones (ejemplar muerto). En avistamientos, se indica en paréntesis el número de ejemplares diferentes observados.

Table 1.-White Stork colonies studied in Navarre and the period (years) during which chicks were ringed. For each colony, details are provided on the number of chicks ringed and the number of sightings (live individual) and recoveries (dead individual). In sightings, the number of different individuals observed is indicated. 
El único caso de reclutamiento se observó en la colonia de Cortes, en donde una cigüeña anillada como pollo en mayo de 2013 se avistó en abril de 2015 en la misma colonia, aunque desconocemos si llegó a criar o no.

\section{Discusión}

Este artículo muestra por primera vez para Navarra datos sobre movimientos, dispersión y uso del hábitat de cigüeñas blancas marcadas como pollos en nido en colonias de cría de la Ribera de Navarra. Aunque de carácter preliminar y descriptivo, debido al reducido número de años de seguimiento y tamaño muestral, se observan algunos patrones interesantes que se discuten a continuación.

En conjunto, un 16\% de los individuos que se marcaron como pollos se avistó/recuperó posteriormente. Este porcentaje es bajo si lo comparamos con el 34\% de avistamientos obtenidos en una población estudiada en la provincia de Córdoba entre 1990 y 2001 ( $n=145$ pollos anillados), cuyas áreas de cría también se situaban próximas a vertederos (ver Tortosa et al., 2002). Una tasa de avistamiento/recuperación baja podría explicarse por una mayor tasa de mortalidad en la población de cigüeñas de Navarra en comparación a otras poblaciones, lo cual, por otro lado, parece poco probable. Aunque es cierto que la tasa de supervivencia puede variar entre poblaciones, y ésta se ve afectada por la edad, siendo mayor la supervivencia en adultos que en juveniles (Schaub et al. 2005). Otra posible explicación sería una probabilidad de avistamiento/recuperación baja debido a una dispersión a zonas no prospectadas, haciendo necesario incrementar el esfuerzo de muestreo, como sugieren los datos de este estudio. Al igual que otros estudios demográficos (e.g, Kanyamibwa et al., 1990), en nuestro trabajo también se registró un descenso de observaciones con la edad de las aves marcadas (esto es, se observaron más jóvenes que aves de edades superiores). Este dato deriva de la alta mortalidad juvenil que resulta en un número comparativamente bajo de ejemplares que llegan a edades superiores. Sin embargo, teniendo en cuenta que la mayoría de las prospecciones se realizaron en vertederos, la reducción del número de observaciones con la edad podría deberse en parte a cambios en el uso del espacio de los individuos marcados. De este modo, podría ser que los ejemplares juveniles, más dependientes de una fuente constante y predecible de alimento (vertederos), con el paso del tiempo vayan adquiriendo experiencia que les permita consumir otras presas en áreas de alimentación no muestreadas.

La mayoría de las aves fue avistada/recuperada a poca distancia (media: 48 km) de su colonia de origen. Esto podría ser debido a la conjunción de diversos factores: (1) gran parte de los avistamientos se obtuvo en el vertedero de Culebrete, en Tudela, que se localiza en las proximidades del área de estudio y marcaje; (2) la mayoría de las observaciones se produjeron durante la época de cría y antes de la migración otoñal, periodo durante el cual muchos juveniles e inmaduros todavía frecuentaban las áreas donde nacieron, antes de emprender movimientos migratorios de larga distancia (Molina \& Del Moral, 2005). 
Probablemente, el uso de vertederos ha tenido un importante papel en el aumento de la población de cigüeñas en Navarra, como ha sucedido en otras zonas de España (Molina \& Del Moral, 2005; Sanz-Aguilar et al., 2015). El uso de los vertederos también se observó para las cigüeñas que más se alejaron de su colonia origen. Esto sugiere que el alimento hallado en vertederos no solo puede tener un papel importante en la productividad de las parejas reproductoras (Tortosa et al., 2002), sino también en el sustento de las aves invernantes y/o migratorias (Sanz-Aguilar et al., 2015; Flack et al., 2016) y en el mantenimiento de tasas altas de supervivencia en juveniles. No obstante, en nuestro estudio se pone de manifiesto la existencia de un mayor esfuerzo de muestreo en vertederos en relación a otro tipo de hábitats, como por ejemplo cultivos o humedales, para los que se conoce la abundante presencia de cigüeñas. Así, es destacable que sólo una cigüeña fuera observada en los arrozales del sur de Navarra, muy frecuentados por la especie cuando éstos están inundados en primavera y verano.

Como en otros casos (Molina \& Del Moral, 2005; Flack et al., 2016), parece que las cigüeñas de origen navarro también usan los vertederos como puntos de parada y ganancia de reservas durante el periodo migratorio. Así, un ejemplar anillado en 2014 se avistó en el vertedero de Almagro (Ciudad Real) en agosto del mismo año y, posteriormente, en junio y julio del año siguiente. Otro ejemplar también anillado en 2014 se avistó en el mismo vertedero en marzo de 2015, coincidiendo con la migración de primavera hacia las áreas de cría. Este ejemplar, además, había sido visto en otoño de 2014 en Culebrete. Ante el previsible cierre de vertederos en toda Europa y la consiguiente desaparición de un recurso trófico que a día de hoy es clave para la especie de estudio, cabría esperar cambios en el patrón de movimientos y dinámica de sus poblaciones. Previsiblemente, la ausencia de recursos tróficos abundantes como los hallados en vertederos podría promover movimientos de mayores distancias, tal y como se ha registrado en otras especies oportunistas (Arizaga et al., 2014).

La mayoría de los avistamientos se realizó durante la primavera y verano, coincidiendo con el periodo de cría y semanas previas a la migración de otoño. El vertedero de Culebrete fue, con diferencia, el lugar donde más avistamientos se obtuvieron, desde marzo hasta septiembre, incluyendo uno más en diciembre. Situado cerca de todas las colonias de estudio, se constata, así, el papel de Culebrete como zona de alimentación de las cigüeñas que crían en el sur de Navarra. Además, el uso de Culebrete parece preferente frente a un vertedero también próximo (Ejea de los Caballeros), en el cual sólo se observaron 3 cigüeñas anilladas en Navarra a pesar de haber invertido un esfuerzo de avistamiento similar a Culebrete. Sin embargo, desconocemos si tal uso es debido a la idiosincrasia de cada vertedero, o a que las cigüeñas prefieren realizar movimientos de menor distancia (o ambas), sobre todo durante el periodo de cría y previo a la migración posnupcial. Culebrete, además, es empleado por un gran número de cigüeñas de origen extranjero, así como por otras aves tales como gaviotas, ardéidas o rapaces que hallan en la basura una fuente de alimento constante y predecible. Por otro lado, en España son cada vez más las cigüeñas adultas que acortan su distancia migratoria o incluso ya no migran, principalmente debido 
a la existencia de vertederos, cultivos o piscifactorías que ofrecen una mayor disponibilidad de comida en invierno (Molina \& Del Moral, 2005; Flack et al., 2016). Aunque de carácter preliminar, debido al escaso tamaño muestral, la observación de un ejemplar nacido en la zona de estudio en Culebrete en diciembre es novedosa para Navarra, y apoyaría la idea de que al menos parte de las cigüeñas del norte peninsular ya no migran y permanecen en la región de cría todo el año. En el futuro deberá estudiarse hasta qué punto este comportamiento está extendido en la población navarra, si varía según clases de edad, o cómo podría cambiar ante el cierre de vertederos.

La principal causa de muerte detectada fue la electrocución en tendidos eléctricos o puntos de alimentación de corriente en vías del tren ("feeder"), en todos los casos individuos jóvenes hallados cerca del nido de nacimiento, como muestran otros estudios (Tobolka, 2014). La causa de muerte por electrocución fue confirmada con las lesiones detectadas en las necropsias realizadas en el Centro de Recuperación de Fauna Salvaje de llundain (Gobierno de Navarra). En concreto, tres de los cuatro ejemplares hallados electrocutados se anillaron en la colonia de Castejón, situada a escasos metros de la estación de tren. Estos resultados ponen de manifiesto la vulnerabilidad de los pollos nacidos próximos a infraestructuras ferroviarias, las cuales a menudo pasan desapercibidas como lugares de riesgo de electrocución o colisión de aves (Ibarrondo \& García, 2012). Un cuarto ejemplar se halló electrocutado bajo una red de distribución en el polígono de Alfaro. Identificar los soportes eléctricos más peligrosos y exigir su corrección o cambio a las compañías responsables reduciría notablemente el número de muertes por electrocución (ver Garrido \& FernándezCruz, 2003; Tintó et al., 2010). Las heridas causadas por cuerdas utilizadas en labores agrícolas (e.g. pacas de paja) fueron la causa de muerte de otro ejemplar. Esta problemática es bien conocida por afectar a numerosas cigüeñas, sobre todo a los pollos durante su fase de desarrollo en el nido. Cabe destacar que durante los marcajes, a menudo se detectaron cuerdas tapizando los nidos de cría, y lo que es peor, enrolladas en las patas y el cuerpo de los pollos (2\% de los casos). Informar y concienciar a agricultores y ganaderos sobre el riesgo de abandonar cuerdas en los campos ayudaría a reducir el problema. Por otro lado, el uso frecuente que las cigüeñas hacen de los vertederos supone a su vez una fuente potencial de contaminantes (Muñoz-Arnanz et al., 2011; de la Casa-Resino et al., 2014). Por ello, sería interesante estudiar el uso que la población navarra hace de los vertederos durante todo el año (ver Gilbert et al., 2016) y los efectos que ello pudiera tener sobre la salud de la población. Finalmente, entre las causas naturales de muerte detectadas en este y otros estudios cabe mencionar la muerte de pollos en nido por inclemencias meteorológias, sobre todo cuando ocurren periodos de fuertes lluvias durante las primeras semanas de vida de los pollos (abril y mayo) (Jovani \& Tella, 2004). Sin embargo, en este estudio no pudimos realizar un seguimiento detallado de cada nido desde que nacen pollos hasta que se anillan (edad a la que ya termorregulan), por lo que no ha sido posible estimar la mortalidad en la población de cigüeñas navarras por inclemencias meteorológicas.

En conclusión, el marcaje de pollos de cigüeña blanca en Navarra aporta por primera vez datos sobre la distribución espacial de individuos pertenecientes a la fracción no repro- 
ductora de la población. Son, no obstante, resultados de carácter preliminar. El gran número de observaciones en vertederos pone de manifiesto el papel de estos enclaves para la especie. No obstante, el esfuerzo de lectura de anillas en otras zonas y hábitats fue considerablemente menor. Por ejemplo, a pesar de que los arrozales del sur de Navarra son muy utilizados por las cigüeñas para alimentarse, tan sólo obtuvimos un avistamiento, mientras que el resto de los avistamientos de la zona se concentraron, exclusivamente, en el vertedero de Culebrete. Por lo tanto, las conclusiones sobre dispersión y uso del hábitat de los ejemplares marcados en este estudio deben tomarse con prudencia debido a que el mayor esfuerzo de muestreo realizado en vertederos probablemente hizo que se sobreestimaran las observaciones en estos lugares frente a otros hábitats. En un futuro, se prevé recopilar más datos que permitan incluir análisis de captura recaptura para poblaciones abiertas, pudiendo así aportar argumentaciones estadísticas a las puramente descriptivas (ver SanzAguilar et al., 2015). Finalmente, sería importante implicar a la sociedad (e.g. naturalistas, ornitólogos), en primer lugar dando a conocer el proyecto y los objetivos del mismo, y en segundo término, promocionando su colaboración a través del seguimiento de ejemplares anillados para incrementar así la tasa de avistamientos/recuperaciones.

\section{Agradecimientos}

Gracias a todas las personas que han enviado información de avistamientos y recuperaciones, en su mayoría a través de las oficinas de anillamiento de la Estación Biológica de Doñana y la Sociedad de Ciencias Aranzadi. Agradecer también a dichas oficinas por el suministro de las anillas, gestión de anillamientos y permisos. Asimismo, agradecemos el envío de toda información sobre anillamientos de cigüeñas en Navarra a las oficinas de la EBD, Aranzadi y SEO/BirdLife (Banco de datos de anillamiento del remite ICONA - "Ministerio de Medio Ambiente, 2016. Datos de anillamiento y recuperaciones en España. Ministerio de Agricultura, Alimentación y Medio Ambiente, SEO/BirdLife, ICO, EBD-CSIC y GOB. Madrid"). Gracias a Gorka Belamendia por toda su ayuda desde el inicio del proyecto, y al Guarderio Forestal del Gobierno de Navarra por su colaboración en labores de marcaje y avistamiento de individuos anillados. Mención especial merecen los integrantes del Grupo de Intervención en altura de Navarra (GIAN): Juan María Barbarin, Pedro Pastor y Oscar Echeverria. Agradecemos al Departamento de Desarrollo Rural, Medio Ambiente y Administración Local del Gobierno de Navarra por los permisos concedidos para el manejo de la especie e intervención del GIAN, así como a párrocos y propietarios por facilitarnos el acceso para las labores de marcaje. Finalmente, agradecer a José l. Aguirre y a un revisor anónimo por sus valiosos comentarios en la revisión del artículo. 


\section{Bibliografía}

- Aguirre, J.I. 2006. Factores que afectan a la supervivencia juvenil de la cigüeña blanca Ciconia ciconia. Tesis doctoral. Universidad Complutense de Madrid. Madrid.

- Arizaga, J., Jover, L., Aldalur, A., Cuadrado, J.F., Herrero, A., Sanpera, C. 2013. Trophic ecology of a resident Yellow-legged Gull (Larus michahellis) population in the Bay of Biscay. Mar. Environ. Res. 87: 19-25.

- Arizaga, J., Aldalur, A., Herrero, A., Cuadrado, J.F., Díez, E., Crespo, A. 2014. Foraging distances of a resident yellow-legged gull (Larus michahellis) population in relation to refuse management on a local scale. Eur. J. Wildl. Res. 60: 171-175.

- Barbraud, C., Barbraud, J.C., Barbraud, M. 1999. Population dynamics of the White Stork Ciconia ciconia in western France. Ibis 141: 469-479.

- Blanco, G. 1996. Population dynamics and communal roosting of White Storks foraging at a Spanish refuse dump. Colon. Waterbird. 19: 273-276.

- De la Casa-Resino, I., Hernández-Moreno, D., Castellano, A., Pérez-López, M., Soler, F. 2014. Breeding near a landfill may influence blood metals ( $\mathrm{Cd}, \mathrm{Pb}, \mathrm{Hg}$, Fe, $\mathrm{Zn}$ ) and metalloids ( $\mathrm{Se}$, As) in white stork (Ciconia ciconia) nestlings. Ecotoxicology 23: 1377-1386.

- Flack, A., Fiedler, W., Blas, J., Pokrovsky, I., Kaatz, M., Mitropolsky, M., Aghababyan, K., Fakriadis, I., Makrigianni, E., Jerzak, L., Azafzaf, H., Feltrup-Azafzaf, C., Rotics, S., Mokotjomela, T.M., Nathan, R., Wikelski, M. 2016. Costs of migratory decisions: A comparison across eight White Stork populations. Sci. Adv. 2: e1500931.

- Garrido, J.R., Fernández-Cruz, M. 2003. Effects of power lines on a White Stork Ciconia ciconia population in central Spain. Ardeola 50: 191-200.

- Gilbert, N.I., Correia, R.A., Silva, J.P., Pacheco, C., Catry, I., Atkinson, P.W., Gill, J.A., Franco, A.M.A. 2016. Are White Storks addicted to junk food? Impacts of landfill use on the movement and behaviour of resident white storks (Ciconia ciconia) from a partially migratory population. Mov Ecol 4:7. doi 10.1186/s40462-016-0070-0

- Gurelur, Fondo Navarro para la Protección del Medio Natural. Campaña Cigüeñas. Censos cigüeñas 2015. http://www.gurelur.org/p/es/proyectos/campana-ciguenas.php

- Ibarrondo, A.G., García, I. 2012. Restocking White Stork "Ciconia ciconia"(L., 1758) population in Biscay: reintroduction in the Urdaibai Biosphere Reserve. Munibe Ciencias naturales 60: 191200.

- Jovani, R., Tella, J.L. 2004. Age-related environmental sensitivity and weather mediated nestling mortality in White Storks Ciconia ciconia. Ecography 27: 611-618.

- Kanyamibwa, S., Schierer, A., Pradel, R., Lebreton, J.D. 1990. Changes in adult annual survival rates in a western European population of the White Stork Ciconia ciconia. Ibis 132: 27-35.

- Molina, B., Del Moral, J.C. 2005. La Cigüeña Blanca en España. VI Censo Internacional (2004). SEO/BirdLife. Madrid.

- Muñoz-Arnanz, J., Sáez, M., Aguirre, J.I., Hiraldo, F., Baos, R., Pacepavicius, G., Alaee, M., Jiménez, B. 2011. Predominance of BDE-209 and other higher brominated diphenyl ethers in eggs of White Stork (Ciconia ciconia) colonies from Spain. Environ. Int. 37: 572-576. 
- Ramos, R., Ramírez, F., Sanpera, C., Jover, L., Ruiz, X. 2009. Diet of Yellow-legged Gull (Larus michahellis) chicks along the Spanish Western Mediterranean coast: the relevance of refuse dumps. J. Ornithol. 150: 265-272.

- Sanz-Aguilar, A., Jovani, R., Melián, C.J., Pradel, R., Tella, J.L. 2015. Multi-event capture-recapture analysis reveals individual foraging specialization in a generalist species. Ecology 96: 1650-1660.

- Schaub, M., Kania, W., Köppen, U. 2005. Variation of primary production during winter induces synchrony in survival rates in migratory white storks Ciconia ciconia. J. Anim. Ecol. 74: 656-666.

- Tintó, A., Real, J., Mañosa, S. 2010. Predicting and correcting electrocution of birds in Mediterranean areas. J. Wildl. Manag. 74: 1852-1862.

- Tobolka, M. 2014. Importance of Juvenile Mortality in Birds' Population: Early Post-Fledging Mortality and Causes of Death in White Stork Ciconia ciconia. Pol. J. Ecol. 62: 807-813.

- Tortosa, F.S., Caballero, J.M., Reyes-López, J. 2002. Effect of rubbish dumps on breeding success in the White Stork in southern Spain. Waterbirds 25: 39-43.

- Vergara, P., Aguirre, J.I., Fernández-Cruz, M. 2004. Fidelidad a los sitios y fenología en la invernada de la cigüeña blanca (Ciconia ciconia) en la Comunidad de Madrid (1998-2002). Anu. Ornitológico Madrid 2003: 74-85. 